\title{
Is there a paradigm shift in use of microsomes and hepatocytes in drug discovery and development?
}

\author{
Sumit Basu, Abdul Naveed Shaik* \\ Center for Pharmacometrics and Systems Pharmacology, Department of Pharmaceutics, University of Florida. Orlando \\ FL. \\ *Corresponding Author: E-mail: naveedshaik@gmail.com; Tel.: +1-407-313-7009.
}

Received: June 13, 2016; Revised: June 20, 2016; Published: June 29, 2016

In last two decades, the field of drug metabolism and pharmacokinetics (DMPK) has been considerably improved due to various reasons, i.e., routine incorporation of DMPK into early drug discovery, better understanding of drug dispositional elements (drug metabolizing enzymes and uptake/efflux transporters) and implementation of optimized DMPK properties in preclinical drug discovery and development. In this regard, both in vitro and in vivo experimental tools play significant role to understand different critical Absorption, Distribution, Metabolism and Elimination (ADME) parameters which accelerated tremendously the lead selection, lead optimization and the preclinical development of the drug candidate prior to the clinical trials. Among these experimental tools, in vitro assays are quite advantageous in comparison to the in vivo models because of its convenient and economical way to address the specific questions in short time period. These in vitro experimental models are often employed to determine the nature of enzyme induction/inhibition (mainly CYPs) in drug-drug interaction (DDI) studies, metabolite identification, reaction phenotyping and elucidation of the biotransformation/metabolic pathway of new chemical entities (NCEs) $[1,2]$. Although it has been shown that due to species related differences, preclinical species cannot always predict human CYP induction/inhibition; nonetheless a good in vitro-in vivo correlation has always been observed when human based in-vitro assays have been employed [3].

In case of metabolite identification and enzyme kinetic studies, the best way to investigate the metabolic profile of the drug in a certain tissue is to procure the tissue specimen and evaluate the activity. In case of liver, this particular method has been applied successfully and undoubtedly this is the method of choice to gain information on hepatic drug metabolism. However, in order to extrapolate the research findings in human, we need to consider additional factors such as ethics, sampling procedure and bioanalysis etc. In addition, although a normal liver is imperative for the research purpose, in reality most of the human liver material is pathological to some extent. Depending on what kind of questions we want to address, a variety of different preparations can be used, such as a fully physiological preparation (e.g. a whole perfused liver) or a strictly biochemical preparation (e.g. cellular sub-fractions such as microsomes) or a compromise situation (e.g. liver slices, cubes and hepatocytes etc.).

Among all the established in vitro experimental tools, microsomes are the mostly widely used 
subcellular fractions for drug metabolism studies as it is inexpensive and easy to handle [4]. Microsomal assays are the default assays for metabolism and DDI studies at the drug discovery stage [5]. In order to determine intrinsic clearance of NCEs, which is a useful parameter for screening process of stable compounds and for establishing an in vitro correlation between animals and humans, microsomal assays play significant role. In addition, this assay can also be utilized to assess extra-hepatic metabolism and determine the DDI potential of the drug candidate in preclinical phase [6]. Apart from liver, microsomes can also be utilized to focus on the region-specific activity of the intestinal enzymes. In general, the experimental conditions utilizing microsomes can be easily controlled in comparison to in vivo and in situ models. Moreover, these methods are theoretically applicable to all species and can be used to study phase I and phase II drug metabolism to get the enzyme isoforms activity profile $[7,8]$. There are few limitations of microsomes, for instance, they have to be supplied with co-factors like NADPH, or UDPGA for the metabolic reactions to initiate, and lack the cell membranes to mimic the physiological environment in hepatocytes $[7,8]$. In addition, it suffers from its non-physiological nature due to the absence of cellular metabolism, membrane transport and production of co-factor $[3,9]$. Furthermore, this tissue preparation cannot provide the information on the activities of the transporters along the intestine, which can be critical for the investigation of gut metabolism to rationalize the absorption and permeability of the compound. The preparations of intestinal microsomes are more prone to artefacts in comparison to the preparation of the liver microsomes.

Several attempts have been made to reach a mid-point between the physiological complexity and biochemical ease. For that effort, liver slices as well as isolated cells have been used. Liver slices have the physiological cell to cell contact needed for the liver preparation but are somewhat unsatisfactory due to the inability to get access of the substrate and nutrient to the centre of the preparation. In comparison, isolated liver cells are regarded as the best alternate, as they can be stored in cultures for extended periods of time without losing their differentiated functions. Due to the presence of various uptake or efflux transporters in hepatocyte cell membranes, this particular subcellular fraction can be used to determine the role of transporter on the disposition of NCE in preclinical discovery phase. The primary culture of hepatocytes provides a physiological environment that closely mimics the in vivo conditions as it contains enzyme and co-factors at physiological concentrations. In drug discovery, hepatocytes are mainly used as sandwich cultures or suspensions which are primarily used for the study of metabolic stability or transporter-mediated uptake of drug candidates. Like microsomes, hepatocytes can also be used in DDI predictions and in determining intrinsic clearance. However, the major application of cultured hepatocytes is in CYP induction studies as enzyme induction may involve gene transcription and translation [10]. Despite hepatocytes playing a significant role in drug discovery, their application is to some extent limited because they are not readily available and difficult to reproduce the results due to high inter individual variability between human liver donors. Of late, freshly isolated, pooled cryopreserved hepatocytes are being used to circumvent this problem which allows researchers to use the same lot of cells over a period of time. However, improving long term culture of hepatocyte has been an area of research focus in the last decade.

In the cell system, drugs never have easy access to the drug metabolizing enzymes. They have to pass through barriers containing drug transporters which facilitate the movement of these drugs across the membrane either concentrating the cell via influx transporters, or dispersing the drug through efflux transporters denying access of enzymes to the drug [11]. Due to the involvement of these drug transporters, in drug disposition and DDIs, there has been major interest in the past decade to study the role of these transporters in drug discovery and development. Hepatocytes have a cellular organization 
containing the necessary co-factors and membranes transporters, which represent a more complete system to study drug metabolism. Due to the high cost and the complexity in conducting experiments with hepatocytes, microsomes still remain the tool of choice for early drug discovery studies. Due to the advancement of technology, commercial companies and research organizations can now obtain human livers, make and store deep frozen slices and isolated hepatocytes in research and drug testing. From the literature studies it is quite evident that instead of following a general method to procure and utilizing the tissue, a case by case approach has always been taken depending on what kind of questions we would like to answer, physiological or biochemical. For an example, in order to measure the change of phase I or II drug metabolizing enzyme content, the usage of microsomes or other subcellular fraction is appropriate. On the other hand, to assess the impact of transporters on the disposition of NCEs in discovery phase as well as to investigate the presence or nature of hepatotoxicity, hepatocytes or whole liver preparation will be more useful. Reverting back to the title of this opinion "Is there a Paradigm Shift in use of Microsomes and Hepatocytes in Drug Discovery and Development?"; based on the literature, even though utilization of hepatocytes in drug discovery and development has been increasing in the last decade, the choice of in vitro tool mainly depends on the type of study performed.

\section{References}

[1] A.S. Kalgutkar, R.S. Obach, T.S. Maurer, Current drug metabolism 8 (2007) 407-447.

[2] A.N. Shaik, T. Bohnert, D.A. Williams, L.L. Gan, B.W. LeDuc, Journal of pharmaceutical sciences 105 (2016) 1976-1986.

[3] R.S. Obach, Drug metabolism and disposition: the biological fate of chemicals 27 (1999) 1350-1359.

[4] D. Zhang, G. Luo, X. Ding, C. Lu, Acta Pharmaceutica Sinica B 2 (2012) 549-561.

[5] A.N. Shaik, R. Grater, M. Lulla, D.A. Williams, L.L. Gan, T. Bohnert, B.W. LeDuc, Journal of chromatography B, Analytical technologies in the biomedical and life sciences 1008 (2016) 164-173.

[6] A.N. Shaik, M.Y. Mahat, K. Kandasamy, B.W. LeDuc, A.A. Khan, AZOLES AS NOVEL INHIBITORS OF MONOAMINE OXIDASE (MAO) A AND B ISOZYMES, Drug metabolism reviews, TAYLOR \& FRANCIS LTD 4 PARK SQUARE, MILTON PARK, ABINGDON OX14 4RN, OXON, ENGLAND, 2015, pp. 204-204.

[7] B. Wu, K. Kulkarni, S. Basu, S. Zhang, M. Hu, Journal of pharmaceutical sciences 100 (2011) 36553681.

[8] B. Wu, S. Basu, S. Meng, X. Wang, M. Hu, Current drug metabolism 12 (2011) 900-916.

[9] D.M. Cross, M.K. Bayliss, Drug metabolism reviews 32 (2000) 219-240.

[10] G. Luo, M. Cunningham, S. Kim, T. Burn, J. Lin, M. Sinz, G. Hamilton, C. Rizzo, S. Jolley, D. Gilbert, A. Downey, D. Mudra, R. Graham, K. Carroll, J. Xie, A. Madan, A. Parkinson, D. Christ, B. Selling, E. LeCluyse, L.S. Gan, Drug metabolism and disposition: the biological fate of chemicals 30 (2002) 795804.

[11] S. Gao, S. Basu, Z. Yang, A. Deb, M. Hu, Current drug targets 13 (2012) 1885-1899.

C 2016 by the authors; licensee IAPC, Zagreb, Croatia. This article is an open-access article distributed under the terms and conditions of the Creative Commons Attribution license (http://creativecommons.org/licenses/by/3.0/) (cc)) EY 